\title{
Host acceptance and life-history traits in Drosophila busckii: tests of the hierarchy-threshold model
}

\author{
Steven P. Courtney and \\ Jeffrey J. Hard
}

Department of Biology, University of Oregon, Eugene, Oregon 97403, U.S.A.

\begin{abstract}
The hierarchy-threshold model for host-choice in insects predicts positive genetic correlations between use of different hosts, and between host use and the number of eggs carried by females. Life history theory predicts negative covariance between reproductive capacity and lifespan; hence host use and lifespan should be negatively correlated. These predictions were tested in half-sib breeding designs with the cosmopolitan Drosophila busckii. The predictions of the hiexarchy-threshold model were met, supporting previous findings with other Drosophila. Predictions from life-history theory were not met for the population studied.
\end{abstract}

\section{INTRODUCTION}

A number of studies have recently documented the presence of genetic variance affecting host choice behaviour within natural populations of plantfeeding insects (Tavormina, 1982; Jaenike and Grimaldi, 1983; Via, 1984; Lofdahl, 1987; Jaenike, 1987; Thompson, 1988a; Courtney and Chen, 1988). In reviewing these and other data, Futuyma and Petersen (1985) have drawn attention to the scarcity of information regarding the factors that maintain such variation within populations. In particular, Futuyma and Petersen point out the need for studies of genetic covariance of other characters, and of the nature of genetic correlations between characters. Genetic correlations due to pleiotropy may impose constraints on the evolution of host choice behaviour. Similarly, correlated responses to natural selection may explain both observed patterns of host use, and the maintenance of additive variance for host selection. Here we test a model which predicts pleiotropic interactions between host use characters, using a population of the cosmopolitan Drosophila busckii.

Courtney et al. (1989) have proposed a comprehensive theory for the mechanistic control of host choice behaviour. The "hierarchy-threshold" model which they advance makes a number of predictions concerning genetic variance for host use, and covariance with other characters. The model shows that pleiotropy between traits is expected to maintain genetic variability. Courtney et al. argue that insects rank potential hosts in order of decreasing acceptability; high ranking hosts are always acceptable, while low-ranking hosts may become acceptable, dependent upon environmental conditions. An insect accepting a low-ranking host must therefore also accept all higher-ranked hosts. The model finds some empirical support in studies by other workers, notably Wiklund $(1981,1982)$, Singer $(1982,1986)$, Lofdahl (1987) and Thompson (1988a). Factors influencing the rate at which hosts become accepted include current eggload borne by females (Fitt, 1986) and adult experience (Jaenike, 1982, 1983). Specific predictions made by Courtney et al. (1989) include: (1) Significant levels of additive genetic variance for use of low-ranking hosts will be maintained in populations, since such variance is rarely subject to direct selection (this holds true even if the host is never experienced in the population in question, i.e., it is a novel host); (2) less variance is expected for use of higher-ranked hosts (unless they are novel) due to direct selection on such traits; (3) significant positive genetic correlations are expected between use of different lowranking, novel hosts; (4) there will be genetic correlations between use of low-ranking hosts and factors influencing acceptance (high eggload increases acceptance-therefore we expect a positive genetic correlation between these traits); (5) no such correlations are expected for characters 
measured against use of high-ranking hosts. These predictions have been met by several studies. Lofdahl (1987) reports additive genetic variance for use of a novel cactus species by $D$. mojavensis; similar results obtain for $D$. suboccidentalis, a mushroom feeder, when presented with two novel hosts, commercial mushrom (Agaricus campestris) and cucumber (Cucumis sativa) (Courtney and Chen, 1988; Chen, 1987). Courtney et al. (1989) report a significant positive genetic correlation between use of low-ranked Cucumis and eggload in 6-day-old $D$. suboccidentalis, but no correlation between use of higher-ranked Agaricus and eggload.

D. suboccidentalis might be seen as an atypical species. It is an ecologically monophagous species (sensu Smiley, 1978), which uses only fungi of genus Ramaria in the study population of the Cascade mountains in western Oregon (Courtney et al., 1989). Here, other fungi are rare and unpredictable, and Ramaria is the only relatively abundant resource. Monophagous populations are, by definition, subject to little direct selection on host choice. Results for D. suboccidentalis might therefore reflect the special circumstances of a monophagous species. The present study was carried out to determine whether similar results may be seen in polyphagous species, which live under more variable selection regimes. The cosmopolitan $D$. busckii was chosen because of its extreme breadth of diet in the wild (many rotting substrates, including mushrooms, are used), and because it is distantly related to $D$. suboccidentalis. $D$. busckii is in subgenus Drosophila; D. suboccidentalis is a member of the quinaria complex of subgenus Dorsilopha. If the predictions of the hierarchythreshold model are met for such ecologically and taxonomically diverse species, the theory may be robust.

An additional interest was to incorporate into our experimental design consideration of another life-history character, adult lifespan. Considerable attention has been given to theories of life-history evolution which rely on antagonistic pleiotrophy between life-history components. For instance, Rose and Charlesworth (1981) show that, in some populations of $D$. melanogaster, early fecundity and lifespan are negatively genetically correlated. Some other studies support this result (e.g., Roach, 1986; Luckinbill et al., 1987; Scheiner et al., 1989) but others find equivocal (Hughes and Clark, 1988) or even opposite results (Giesel et al., 1982). Whatever the generality of the results, antagonistic pleiotropy between fecundity and lifespan could have important ramifications given that fecundity itself is predicted to covary with host use. A deriva- tive prediction of life-history theory and the hierarchy-threshold model is thus that we expect negative covariance between lifespan and acceptance of low-ranking hosts, due to pleiotropic effects through reproductive allocation.

\section{METHODS}

We carried out a standard half-sib breeding design using tenth generation descendants of $D$. busckii caught at Eugene, Oregon on mushroom baits. Stocks were maintained at high population numbers $(>1000)$ over Carolina instant Drosophila medium at $25^{\circ} \mathrm{C}$, and under constant light. In all, 23 males were successfully mated to an average of $5 \cdot 5$ females, producing 126 full-sib families. Families were raised on instant medium as before. Half-sib families were distributed randomly among vials and with respect to position to the rearing chamber and time of onset. This procedure ensures that common environment (larval rearing media) effects did not influence the estimates of heritabiliy or genetic correlations, derived from half-sib designs (Falconer, 1981). Flies were sexed at emergence, and the sexes separated. Females were then divided among four treatments. One group was dissected at age 6 days, to determine the eggload carried. Eggload is measured as an index of reproductive allocation, independent of mating history. Eggload was measured in virgins, because mated females would begin to lay eggs. This seems a reasonable way to study host choice, since females mate at the oviposition site, and must therefore sometimes approach hosts as virgins. A second group was maintained (virgin) over medium, and the lifespan of each individual recorded. The remaining flies were mated at day 6 and exposed for 24 hours to approximately $1 \mathrm{~g}$ of fresh material of one of two hosts: A. campestris or C. sativa. Oviposition substrates were then examined for the presence of eggs; if one egg or more was found, then the host was scored as accepted. The total number of eggs laid on each host was also recorded. Only one host was offered to each fly; This procedure is necessary because exposure to a host changes the behaviour of females on other hosts (Courtney and Gardner unpublished) and may alter both fecundity and survivorship.

Heritabilities of the numbers of eggs laid on either host, of female lifespan and of eggload were estimated using standard methods employing nested ANOVA (Falconer, 1981). Heritabilities of host acceptance were calculated using methods applicable to threshold traits (Robertson and Lerner, 1949); standard errors for unbalanced designs are not calculable, and significance of these 
threshold characters were estimated from $\chi^{2}$ analysis. Genetic correlations were estimated from correlations across family means, following the suggestions of Via (1984) for situations where only one character can be measured per individual. This is a conservative procedure which, relative to other measures of $r_{A}$, tends to underestimate correlations of characters; it does have the advantage of employing standard tests of significance. Percentages were arcsine square-root transformed before analysis.

\section{RESULTS}

Heritabilities of the characters studied are shown in table 1 . As predicted, acceptance of the lowranking host, cucumber, showed significant additive genetic variance, even though this host must be very rarely encountered and used in the field. Heritability of acceptance of the high-ranking host, A. campestris, was not significantly different from zero, again as predicted from the hierarchythreshold model. Heritabilities of the numbers of eggs laid on either host and of life-history characteristics were low, and only that of eggload was significantly different from zero.

A significant positive genetic correlation was found between acceptance of the two hosts (table 2). Acceptance of the low-ranking host was also positively correlated with eggload. The num-

Table 1 Heritabilities of traits in the D. busckii population

\begin{tabular}{llllll}
\hline & & $h^{2}$ & S.E. & $\chi^{2}$ & $P$ \\
\hline E. Eggload & $0 \cdot 24$ & $0 \cdot 11$ & - & - \\
L. Longevity & $0 \cdot 16$ & $0 \cdot 18$ & - & - \\
Ac Acceptance of Cucumis & $0 \cdot 25$ & & $42 \cdot 5$ & $0 \cdot 01$ \\
Aa Acceptance of Agaricus & 0.08 & & $29 \cdot 3$ & ns
\end{tabular}

Acceptance traits were treated as threshold characters, for which no standard errors are calculable for unbalanced data sets; significance levels are determined by $\chi^{2}$ statistic for these traits. bers of eggs laid on the two hosts were not however correlated, although each did weakly correlate with eggload. We interpret these results as supporting the hierarchy-threshold model. Acceptance (whether or not at least one egg is laid) is not closely associated with the numbers of eggs laid by accepting females. This argues that acceptance, and the numbers of eggs laid following acceptance are largely independent characters in D. busckii. Acceptance of the low-ranking host is positively correlated with eggload as predicted. Use of the high-ranking host is not correlated with eggload, again as predicted. Correlations between eggload and the number of eggs laid on either host probably reflect rather simple effects of the number of eggs available for laying.

Predictions based on expectations of antagonistic pleiotropy between lifespan and eggload were not met (table 2). No significant relationship between these two characters was found. Neither did acceptance of cucumber correlate negatively with lifespan. Eggload may not be the most appropriate measure of reproductive allocation; nevertheless, significant additive genetic variance was detected for this trait, indicating the possibility of detecting significant correlations. An unexpected result was the significant positive correlation between lifespan and acceptance of the highranking host. Possible interpretations include: (1) the widespread presence of deleterious mutations in inbred lines, leading to positive correlations between life-history components (Rose, 1984). We reject this interpretation because our stocks were recently derived from natural populations, and were kept at high numbers. Also, no correlation between lifespan and eggload was seen, as predicted from this explanation. (2) Interaction between behaviours favouring survival and those promoting acceptance of a normal host. This might for instance occur where adults are attracted to feed on the oviposition substrate. We do not favour this hypothesis, and currently lack a satisfactory explanation for the observed correlation.

Table 2 Genetic correlations between traits, as described in the text. $n=23$ in all cases

\begin{tabular}{llcrrrl} 
& & L. & Ac & Aa & Nc & Na \\
\hline E. Eggload & -0.038 & $0.628^{* * *}$ & 0.234 & $0.466^{*}$ & $0.437^{*}$ \\
L. Longevity & & -0.036 & $0.527^{* * *}$ & -0.132 & 0.330 \\
Ac & Acceptance of Cucumis & & & $0.552^{* *}$ & 0.125 & \\
Aa Acceptance of Agaricus & & & & & 0.290 \\
$\mathrm{Nc}$ Number of eggs on Cucumis & & & & & 0.151 \\
$\mathrm{Na}$ & Number of eggs on Agaricus & & & & & \\
\hline
\end{tabular}

Significance levels $P<0.05^{*}, 0.01^{* *}, 0.005^{* * *}$. Full-sib correlations did not yield additional information, except that the number of eggs laid by sibs on the two hosts were significantly correlated $(r=0.452, P<0.01)$ 


\section{DISCUSSION}

The predictions of the hiearchy-threshold model (Courtney et al., 1989) have been met for D. busckii. Acceptance of hosts does appear to depend on physiological status of the female. Observed patterns of genetic correlations imply pleiotropy, since it is unlikely that selection in the uniform lab environment would maintain sufficient levels of linkage disequilibrium to explain such high genetic correlations. All observations therefore support the proposition of Courtney et al., that host choice is strongly affected by internal factors, and that pleiotropy between characters may underlie and help to explain the observed high levels of genetic variance for host acceptance behaviour.

Our results for D. busckii confirm earlier, similar findings in $D$. suboccidentalis. These two species are at opposite ends of the resourcebreadth continuum, since $D$. suboccidentalis utilises a narrow range of hosts (so far we have found it only on Ramaria) while $D$. busckii uses many substrates including mushrooms, skunk cabbage, rotting wood and garbage. These two species are also taxonomically distant, occurring in different subgenera of Drosophila. That the underlying genetic structure of the two species is so similar argues that the hierarchy-threshold model is not sensitive to taxonomic and ecological considerations, and may be generally applicable.

The exact values for $h^{2}$ and $r_{A}$ in any lab study must always be treated with caution. Nevertheless our results are in broad agreement with earlier results for $D$. mojavensis and $D$. suboccidentalis where heritability of acceptance of novel hosts was low even if significant (12-22 per cent) (Lofdahl, 1987; Courtney and Chen, 1988; Courtney et al., 1989). Correlations between characters were relatively high compared to other behavioural studies (Roff and Mousseau, 1987) but comparable to our earlier results for $D$. suboccidentalis. This again supports our mechanistic explanation that correlations result from the constraint implied by physiological processes affecting both egg maturation and host choice.

An additional life-history component, female longevity, was not correlated with host choice in the manner predicted. This suggests that selection could operate on variation affecting longevity independently from selection acting on variation affecting host acceptance. Nevertheless these characters may show some association. Long-lived flies will tend to have more opportunities for acceptance or rejection of hosts. The hierarchythreshold model also suggests that animals accept lower-ranking hosts following host deprivation (e.g., after periods of inclement weather, when dispersal is prevented, or when host abundance is low). Longer-lived flies may well then be the part of the population most likely to survive inter-host periods, and hence to be subject to selection for acceptance of lower-ranked hosts. A priori then, we may expect positive genetic correlations between longevity and acceptance of low-ranked hosts, due to linkage disequilibrium. We should not however detect this pattern (expected under field conditions) in our lab-reared populations unless linkage is very strong.

Our results indicate the presence within populations of $D$. busckii of genetic variance for use of a novel host Cucumis. If ecological conditions ever arose where use of this host was favoured, this genetic variance would already be present in the population, and available for the operation of selection. As pointed out by Lofdahl (1987) and Courtney et al. (1989), the presence of genetic variance for use of novel hosts implies that host shifts may occur rapidly. What maintains this variance? Our results clearly implicate correlated selection on reproductive allocation as one plausible explanation. If eggload early in life is subject to fluctuating selection, we may expect genetic variance for this character to be maintained. Given the variable thermal environment and patchy host distribution of both $D$. busckii and D. suboccidentalis, we feel that further attention should be given to effects on female eggload.

Our results also suggest that selection on host use may result in responses in other characters. In particular, selection against use of suboptimal hosts may initially result in lowered early fecundity. However we believe that such selection against a fitness component would result in a change in the correlation between the traits; in this case the internal threshold for host acceptance would rise, and the correlation would weaken. Selection on acceptance of one host will also have correlated responses on acceptance of other hosts. If our population of $D$. busckii had experienced strong directional selection for use of Cucumis we would also expect the population to readily accept Agaricus and other hosts ranked higher than Cucumis. This effect has potential importance in insect pests, where colonists onto one novel host (crop) are also likely to be colonists of other hosts.

Ourstudies have not addressed the relationship of adult and larval characters. In particular we have not considered here the suitability of different hosts for larval development. Both Cucumis and Agaricus are well able to support larval growth of 
D. busckii. Generally, adult oviposition range of insects is less broad than larval dietary tolerance (Wiklund, 1975). We agree with Futuyma (1983), Jermy (1984) and others that understanding oviposition choice is therefore critical to our knowledge of the evolution of host use. The hierarchythreshold model, which concerns the mechanism of oviposition choice, implies that hosts are ranked in order of acceptability. The evolutionary relationship between acceptability and suitability for larval development is largely unexplored (Thompson, 1988b, but see Rausher, 1984; Rausher and Englander, 1988). Given that selection against use of low-ranking hosts will usually be both weak and sporadic, the exact relationship of adult preference and performance requires much further investigation.

Acknowledgements We thank Ian Fisher and Alex Gardner for help with laboratory work. W. Bradshaw and L. Heisler have made useful comments on our ideas.

\section{REFERENCES}

CHEN, G. K. 1987. Oviposition behavior of Drosophila suboccidentalis. M.Sc. Thesis, University of Oregon.

COURTNEY, S. P. AND CHEN, G. K. 1988. Genetic and environmental variation in oviposition behaviour in the mycophagous Drosophila suboccidentalis Spcr. Func. Ecol., 2, 521-528.

COURTNEY, S. P., GARDNER, A. AND CHEN, G. K. 1989. A general model for individual host selection. Oikos, 55 , $55-65$.

FALCONER, D. S. 1981. An Introduction to Quantitative Genetics, 2nd edn, Longman, New York.

FITT, G. P. 1986. The influence of a shortage of hosts on the specificity of oviposition behavior in species of Dacus (Diptera: Tephritidae). Physiol. Entomol., 11, 133-143.

FUTUYMA, D. J. 1983. Selective factors in the evolution of host choice by phytophagous insects. In Ahmad, S. (ed.) Herbivorous Insects: Host-Seeking Behavior and Mechanisms, Academic Press, New York, pp. 227-244.

FUTUYMA, D. J. AND PETERSON, S. C. 1985. Genetic variation in the use of resources by insects. Ann. Rev. Entomol., 30, 217-238.

GIESEL, J. T., MURPHY, P. A. AND MANLOVE, M. N. 1982. The influence of temperature on genetic interrelationships of life history traits in a population of Drosophila melanogaster: what tangled data sets we weave. Am. Nat., $119,464-479$.

HUGHES, D. M. AND CLARK, A. G. 1988. Analysis of the genetic structure of life history of Drosophila melanogaster using recombinant extracted lines. Evolution, 47, 1309-1320.

JAENIKE, J. 1982. Environmental modification of oviposition behaviour in Drosophila. Am. Nat., 119, 784-802.

JAENIKE, J. 1983. Induction of host preference in Drosophila melanogaster. Oecologia, 58, 320-325.

JAENIKE, J. 1987. Genetics of oviposition-site preference in Drosophila tripunctata. Heredity, 59, 363-369.
JAENIKE, J. AND GRIMALDI, D. 1983. Genetic variance for host preference within and among populations of Drosophila tripunctata. Evolution, 37, 1023-1033.

JERMY, T. 1984. Evolution of insect/hostplant relationships. Am. Nat., 124, 609-630.

LOFDAHL, K. 1987. A genetic analysis of habitat selection in the cactophilic species, Drosophila mojavensis. In Huettel, M. D. (ed.) Evolutionary Genetics of Invertebrate Behavior, Plenum Press, New York, pp. 153-162.

LUCKINBILL, L. S., CLARE, M. J., KRELL, W. L., CIROCCO, W. C. AND RICHARDS, P. A. 1987. Estimating the number of genetic elements that defer senescence in Drosophila. Evol. Ecol., 1, 37-46.

RAUSHER, M. D. 1984. Tradeoffs in performance on different hosts: Evidence from within- and between-site variation in the beetle Deloyala guttata. Evolution, 38, 582-595.

RAUSHER, M. D. AND ENGLANDER, R. 1987. The evolution of habitat preference. II. Evolutionary genetic stability under soft selection. Theor. Pop. Biol., 31, 116-139.

ROACH, D. 1986. Life-history variation in Geranium carolinianum. 1. Covariation between characters at different stages of the life cycle. Am. Nat., 128, 47-57.

ROBERTSON, A. AND LERNER, M. 1949. The heritability of all-or-none traits: viability of poultry. Genetics, 34, 395411.

ROFF, D. AND MOUSSEAU, T. A. 1987 Quantitative genetics and fitness: lessons from Drosophila. Heredity, 58, 103-118.

ROSE, M. 1984. Genetic covariation in Drosophila life history: untangling the data. Am. Nat., 123, 565-569.

ROSE, M. R. AND C.HARLESWORTH, B. 1981. Genetics of life history in Drosophila melanogaster. I. sib analysis of adult females. Genetics, 97, 173-186.

SCHEINER, S. M., CAPLAN, R. L., LYMAN, R. F. 1989. A search for trade-offs among life history traits in Drosophila melanogaster. Evol. Ecol, 3, 51-63.

SINGER, M. C. 1982. Determinants of multiple host use by a phytophagous insect population. Evolution, 37, 389-403.

SINGER, M. C. 1986 The definition and measurement of oviposition preference in plant-feeding insects. In Miller, J. and Miller, T. A. (eds) Methods for Studying Mechanistic Interactions Between Insects and Plants. Springer-Verlag, Berlin, 1987

SMILEY, J. T. 1978. Plant chemistry and the evolution of host specificity: New evidence from Heliconius and Passiflora. Science, 201, 745-747.

TAVORMINA, S. J. 1982. Sympatric genetic divergence in the leaf-mining insect Liriomyza brassicae (Diptera: Agromyzidae) Evolution, 36, 523-534.

THOMPSON, J. N. 1988a. Variation in preference and specificity in monophagous and oligophagous swallowtail butterflies. Evolution, 42, 118-128

THOMPSON, J. N. $1988 b$. Evolutionary ecology of the relationship between oviposition preference and performance of offspring in phytophagous insects. Entomol. Exp. Appl., 47, 3-14.

VIA, S. 1984. Quantitative genetics of polyphagy in an insect herbivore, 1. Genotype-environment interaction in larval performance on different hostplant species. Evolution, 38, $881-895$

WIKLUND, C. 1981. Generalist vs. specialist oviposition behavior in Papilio machaon (Lepidoptera) and functional aspects of the hierarchy of oviposition preferences. Oikos, $36,163-170$.

WIKLUND, C. 1982. Generalist versus specialist utilization of host plants among butterflies. Proc. Int. Symp. Insect-Plant Relationships, 5, 181-191. 